\title{
Editorial
}

\section{Message from the new Editor-in-Chief}

\author{
Eddy Lelièvre-Berna \\ Services for Advanced Neutron Environments, Institut Laue-Langevin, Grenoble, France \\ E-mail:lelievre@ill.eu
}

This is a great honour to succeed to Gerry Lander and Colin Carlile. I met Gerry more than two decades ago on the polarised neutron diffractometer D3 at the Institut Laue-Langevin while I was his local contact, and Colin a few years later when he took the position of Associate Director before becoming Director of the ILL. I have learnt a lot from them as from other colleagues like P. Jane Brown, S. Pujol and F. Tasset. I owe them a lot. As Albert Einstein wrote in "My Credo" at the end of August 1932: "I am often troubled by the thought that my life is based to such a large extent on the work of my fellow human beings, and I am aware of my great indebtedness to them."

This is also a challenge to succeed to Gerry and Colin. The neutron community is spread over the world but small. We are overbooked and pushed to publish excellence. At the same time, a young generation of technicians, engineers and scientists does its best to build new sources or start new facilities. This is a very positive trend but also very challenging. To help them succeed and elevate the role of neutrons in Science, the minimum we, the community, must do, is to humbly share our know-how and bring state-of-the-art solutions to (almost) known problems.

The Journal of Neutron Research is the only journal dedicated to neutron scattering and connecting several generations of neutron experts. We find treasures in the archives, from early evaluations of future long pulse spallation sources to industrial applications of neutrons without forgetting regular and review articles or proceedings of workshops on neutron techniques.

To my point of view, the future of the neutron community depends on our capability to progress and innovate together. My wish is that JNR becomes the standard platform for exchanging expertise in measuring capabilities, hardware and software, without forgetting neutron sources, moderators and guides. With your contribution, JNR can even be transformed into an incubator of ideas which will amplify ambitions and finally leverage the construction of unprecedented neutron sources.

JNR is your resource and I count on you to make this happen. With the Editorial Board, David Greaves and Kairi Look from IOS Press, we shall do our best to facilitate the submission process.

Thank you.

Editor-in-Chief, Journal of Neutron Research
Eddy Lelièvre-Berna
Services for Advanced Neutron Environments
Institut Laue-Langevin
71 Avenue des Martyrs, CS 20156
38042 Grenoble Cedex 9
France
E-mail: lelievre@ill.eu

